\title{
Manifestações clínicas da Leishmaniose Cutânea no paciente com HIV
}

\author{
Clinical manifestations of Cutaneous Leishmaniasis in HIV
}

Manifestaciones clínicas de la Leishmaniasis Cutánea en el HIV

\begin{abstract}
Danyella Oliveira de Paula ${ }^{1 *}$, Ana Clara Borsari Serra ${ }^{2}$, Anna Carolina Teixeira Lengruber Amaral ${ }^{3}$, Júlia de Laura Calmon Vasconcelos ${ }^{4}$, Luiza Oliveira Coelho ${ }^{5}$, Maria Eduarda de Carvalho Brêda ${ }^{6}$, Mariana Vieira Martins de Mello ${ }^{7}$, Natália Vargas do Nascimento ${ }^{8}$, Vitória de Lima Fujinami Tano ${ }^{9}$, Marina Carrara Camillo Barbosa ${ }^{10}$.
\end{abstract}

\section{RESUMO}

Objetivo: Revisar como se comporta a coinfecção do Vírus da Imunodeficiência Humana (HIV)/Leishmaniose, bem como suas características clínicas. Revisão bibliográfica: A leishmaniose cutânea é uma doença endêmica não contagiosa, causada por protozoários do gênero Leishmania que acometem pele e mucosas. A transmissão ocorre através da picada de insetos infectados. Por outro lado, a Síndrome da Imunodeficiência Adquirida é caracterizada pela destruição de linfócitos CD4, prejudicando a imunidade celular do organismo e gerando espaço para infecções oportunistas, como a Leishmaniose cutânea. Por conseguinte, a manifestação clínica da coinfecção de HIV/Leishmaniose ocorre diferente da manifestação da leishmaniose, pois há o aparecimento de características clínicas atípicas sendo o acometimento do trato gastrointestinal e do respiratório, além da baixa contagem de linfócitos CD4+. Considerações finais: A partir dos estudos, foi possível inferir que a coinfecção HIV/Leishmaniose tem um grande efeito prejudicial ao sistema imune, além de apresentarem dificuldades ao tratamento por não haver uma resposta eficaz imunológica.

Palavras-chave: Leishmaniose, Coinfecção, HIV.

\begin{abstract}
Objective: Review how is the behavior of the coinfection Human Immunodeficiency Virus (HIV)/Leishmaniose, as well as clinical features. Bibliographic review: The Leishmaniasis Cutaneous is an endemic disease not contagious, caused by protozoa of the genus Leishmania that affect skin and mucous membranes. The transmission happens through infected insects bite. On the other hand, Acquired Immunodeficiency Syndrome is characterized by the destruction of CD4 lymphocytes, impairing the body's cellular immunity and engendering space for opportunists infections, like Leishmaniasis Cutaneous. Therefore, the clinical manifestation of the coinfection HIV/Leishmaniasis occurs differently than the leishmaniasis manifestation, because there is the appearance of atypical clinical features like the involvement of the gastrointestinal and respiratory tract, in addition to the low CD4 + lymphocyte count. Final considerations: From the studies, it was possible to infer that the coinfection HIV/Leishmaniasis has a big detrimental effect on the immune system, besides presenting difficulties to the treatment difficulties in treatment because there is no effective immune response.
\end{abstract}

Keywords: Leishmaniasis, Coinfection, HIV.

${ }^{1}$ Centro Universitário do Planalto Central Apparecido dos Santos (UNICEPLAC), Brasília - DF.

*E-mail: dany-lella@hotmail.com

2 Universidade Cesumar (UNICESUMAR), Maringá - PR.

${ }^{3}$ Centro Universitário de Brasília (UNICEUB), Brasília - DF.

${ }^{4}$ Faculdade de Minas (FAMINAS), Belo Horizonte - MG.

5 Universidade Nove de Julho (UNINOVE), São Paulo - SP.

${ }^{6}$ Centro Universitário Cesmasc (CESMAC), Maceió - AL.

7 Universidade Potiguar (UNP), Natal - RN.

8 Universidade Católica de Brasília (UCB), Brasília - DF.

${ }^{9}$ Faculdade Ceres (FACERES), São José do Rio Preto - SP.

10 Universidade Metropolitana de Santos (UNIMES), Santos - SP. 


\section{RESUMEN}

Objetivo: Revisar cómo se comporta la coinfección Virus de Inmunodeficiencia Humana (HIV)/Leishmaniasis, así como sus características clínicas. Revisión de literatura: La leishmaniasis cutánea es una enfermedad endémica no contagiosa, causada por protozoos del género Leishmania que afectan la piel y las mucosas. La transmisión se sucede por la picadura de insectos infectados. Por otro lado, el Síndrome de Inmunodeficiencia Adquirida se caracteriza por la destrucción de los linfocitos CD4, perjudicando la inmunidad celular del cuerpo y creando espacio para infecciones oportunistas, como Leishmaniasis cutánea. Por lo tanto, la manifestación clínica de la coinfección por HIV/Leishmaniasis ocurre de manera diferente a la manifestación de leishmaniasis, debido a la aparición de características clínicas atípicas, siendo la afectación del tracto gastrointestinal y respiratorio, además del bajo recuento de linfocitos CD4+. Consideraciones finales: A partir de los estudios, fue posible inferir que el HIV/Leishmaniasis coinfección tiene un gran efecto perjudicial sobre el sistema inmunológico, además de presentar dificultades en el tratamiento porque no hay una respuesta inmunitaria eficaz.

Palabras clave: Leishmaniasis, Coinfección, VIH.

\section{INTRODUÇÃO}

A leishmaniose cutânea é uma doença endêmica no Brasil e acomete anualmente cerca de 1 milhão de pessoas mundialmente. É uma zoonose que possui como vetores cães, roedores, lobos e raposas e humanos, como hospedeiros incidentais; é transmitida pela picada de flebotomíneos, parasita intracelular obrigatório do subgênero leishmania parasites leishmania. A sintomatologia da leishmaniose pode variar de infecção assintomática à letal, e sua apresentação se dá pelo modo visceral ou tegumentar (MARTINEZ DY, et al., 2018).

No que tange a apresentação clínica das lesões tegumentares, essas podem ser únicas ou múltiplas com nódulos eritematosos, pápulas e evoluir para úlceras. Esse quadro pode mimetizar algumas outras patologias, o que dificulta o diagnóstico e leva à submissão de pacientes a tratamentos desnecessários, piorando o quadro clínico e contribuindo para a cadeia de transmissão do agente (MARTINS GAS e LIMA MD, 2013).

Atualmente, existem mais de 30 milhões de pessoas infectadas no mundo pelo Vírus da Imunodeficiência Humana (HIV), destas, mais de 300.000 casos foram registrados no Brasil, o que caracteriza a doença como um problema de saúde não apenas nacional, mas também em nível global. A infecção pelo HIV é caracterizada pela destruição de linfócitos CD4, prejudicando a imunidade celular do organismo e gerando espaço para infecções oportunistas. A transmissão ocorre por meio de contato de secreções fisiológicas, como: saliva, sangue ou sêmen, que contenham vírions HIV livres ou células infectadas (SALOMÃO R, 2017).

No que se diz respeito às manifestações clínicas da coinfecção da leishmaniose com o Vírus da Imunodeficiência Humana (LV-HIV), ela ocorre diferente daquelas sem coinfecção, sendo um exemplo parasitas que ficam isolados em locais em comum. $\mathrm{O}$ aparecimento de características clínicas atípicas seria evidenciado pelo acometimento do trato gastrointestinal e do respiratório, a qual podem ser encontrados em pacientes que possuem baixa contagem de linfócitos CD4+ (SILVA LRM, 2018).

Em resumo, para diagnosticar uma lesão atípica de forma adequada, o foco deve estar no histórico médico e na origem do paciente, pois, clinicamente, as lesões cutâneas localizadas podem se assemelhar a outras patologias de pele, como: blastomicose, esporotricose, pele fúngica diversas infecções, antraz cutâneo, eczema, lepra lepromatosa, tuberculose losis, infecções por Mycobacterium marinum, células basais e escamosas carcinomas (SCC) e picadas de insetos infectados. Comparando-os à história natural da leishmaniose e sempre lembrando de possíveis apresentações atípicas, para então, começar a procurar os melhores métodos de diagnósticos e o tratamento ideal. Assim, avaliando e controlando o progresso da doença (MEIRELES CB, et al., 2017).

Mudanças recentes no quadro epidemiológico da coinfecção do HIV e leishmaniose podem levar a uma sobreposição geográfica, sendo consequência de uma urbanização das leishmanioses e uma interiorização dos quadros de infecção por HIV (SILVA LRM, 2018). As particularidades dos casos de coinfecção HIV/Leishmaniose possuem como características: alta taxa de recidiva, sintomas leves e persistentes e uma proliferação parasitológica mesmo com o tratamento adequado (COTA GF, 2017). 
Ante ao exposto, define-se como objetivo deste trabalho revisar como se comporta a coinfecção de HIV/Leishmaniose, bem como suas características clínicas, uma vez que ambas as infecções são de grande importância mundial e endêmicas no nosso país.

\section{REVISÃO BIBLIOGRÁFICA}

A Leishmaniose é uma doença causada pelo parasita Leishmania, que é um protozoário intracelular, membro da ordem Kinetoplastida, família Trypanosomatidae e possui dois subgêneros: Leishmania (Leishmania) e Leishmania (Viannia) (MARTINS GAS e LIMA MD, 2013). A doença é transmitida por meio de vetores flebotomíneos infectados (fêmeas do mosquito-pólvora) e possui um amplo espectro de manifestações clínicas, variando de acordo com a espécie de Leishmania envolvida. Em raras ocasiões, a transmissão pode ocorrer de forma congênita ou como resultado de transfusão de sangue (SINGH S, et al., 2014).

A leishmaniose é endêmica em mais de 98 países, afetando principalmente a população de baixa renda, desnutridos, moradias precárias, baixa imunidade e falta de recursos financeiros (LINDOSO JAL, et al., 2016). Nas áreas endêmicas, é imprescindível conhecimento das lesões típicas e atípicas pelos médicos, principalmente a forma crônica da LT (MEIRELES CB, et al., 2017).

Ainda mais, essa é uma doença infecciosa zoonótica, amplamente distribuída em todo mundo, desde a Ásia até à América. A taxa de mortalidade associada a essa doença tornou um caso de saúde pública em pelo menos 88 países, e a Organização Mundial da Saúde (OMS) estima que 10 milhões de pessoas sofrem de lesões cutâneas por leishmaniose (MEIRELES CB, et al., 2017).

A Leishmania cursa com três formas clínicas principais de infecção, que são: a Leishmaniose Cutânea (LC), Leishmaniose Mucocutânea (LMC) e Leishmaniose Visceral (LV) (LEELAYOOVA S, et al., 2017).

As diversas manifestações clínicas da Leishmaniose se dão por consequência da interação parasito/hospedeiro, considerada complexa e sem padrão. Essas expressões clínicas podem variar na Leishmaniose Tegumentar (LT), podem ocorrer manifestações assintomáticas, lesões cutâneas, úlceras mucocutâneas e também formas difusas que possuem difícil tratamento (MARTINS GAS e LIMA MD, 2013).

As lesões atípicas da LT foram encontradas nas maiorias dos países endêmicos e não possuem nenhuma relação com idade e sexo do paciente. Essas podem surgir em diversos locais, por exemplo, face, bochechas, orelhas, nariz, pálpebra, membros, tronco, nádegas, palma das mãos e genitálias. São consideradas lesões atípicas por simularem alterações de outras doenças e são denominadas como: aspectos lupóide, eczematoso, erisipeloide, verru-cuscuz, seco, zosteriforme, paroníquico, esporotricóide, chacriforme, úlcera vulcânica anular e eritematosa. Podem também ocorrer lesões semelhantes a micose subcutânea e profunda, doenças malignas como linfoma, pseudolinfoma, carcinomas de células basais e escamosas (MEIRELES CB, et al., 2017).

A transmissão da doença está relacionada a mudanças ambientais, como desmatamento, construção de barragens, sistema de irrigação e urbanização. A cada ano ocorrem aproximadamente 50 a 90 mil novos casos de Leishmaniose Visceral (LV), sendo que mais de 90\% dos casos de LV ocorrem em sete países no mundo: Brasil, Etiópia, Índia, Quênia, Somália, Sudão do Sul e Sudão. Na América Latina, 96\% da LV ocorre no Brasil. Já os casos de LT estão localizados 70-75\% nos países: Afeganistão, Argélia, Brasil, Colômbia, Costa Rica, Etiópia, Irã, Peru, Sudão e Síria (SILVA LRM, et al., 2018; LINDOSO JAL, et al., 2016).

Algumas manifestações frequentes na doença são: alterações dermatológicas, onicogrifose, linfadenopatia, esplenomegalia, hiporexia e apatia. As lesões dermatológicas ocorrem na maioria dos casos e podem se manifestar desde alopecia focal ou generalizada até lesões crostosas e descamação furfurácea (MARTINS GAS e LIMA MD, 2013).

Além disso, as lesões podem aparecer semanas, meses ou até mesmo anos depois da infecção. As lesões primárias podem se expressar sozinhas ou acompanhadas, de forma que pode ocorrer também 0 comprometimento linfático o qual se manifesta como linfadenite ou linfadenopatia, situação essa que é comum 
quando a infecção é causada pelo subgênero Viannia. É importante ressaltar que no Brasil, as infecções se manifestam de maneira assintomática e as formas leves da doença são mais frequentes. $O$ acompanhamento longitudinal do paciente tem apontado que alguns indivíduos persistem assintomáticos ou são capazes de se recuperar espontaneamente em quadros de doença leve, em contrapartida outros em mesma condição acabam por desenvolver a LV (WHO, 2010).

Há inúmeros fatores de risco para o desenvolvimento da LV como: HIV, idade jovem e migração de pessoas não imunes para áreas de endemia, desnutrição e moradia precária. Em pacientes com a LV, cerca de $20-40 \%$ possuem coinfecção LV-HIV (ABONGOMERA C, et al., 2017).

O diagnóstico da leishmaniose é realizado por principalmente três exames: exames parasitológicos, imunológicos e moleculares. O exame parasitológico inicialmente consiste na pesquisa microscópica das formas amastigotas em material obtido da borda da lesão, posteriormente à identificação da espécie de Leishmania spp, ocorre o isolamento in vivo através da inoculação em animais (MARTINS GAS e LIMA MD, 2013).

A respeito dos exames imunológicos a princípio realiza-se o teste de intradermorreação de Montenegro (IDRM) ou da leishmania, esperando-se uma resposta de hipersensibilidade celular retardada, logo após realiza-se testes sorológicos para detecção de anticorpos anti-Leishmania circulantes no soro dos pacientes. Nos exames moleculares realizam análises moleculares do DNA do parasito pela técnica de amplificação por meio da Reação da Cadeia da Polimerase (PCR) (MARTINS GAS e LIMA MD, 2013).

Depois da epidemia de HIV, muitos países constataram coinfecções de Leishmania com o HIV. A relação Leishmania/HIV ocorre devido à superposição geográfica das patologias, da urbanização da leishmaniose e da interiorização da infecção pelo HIV (SINGH S, et al., 2014). Por serem doenças cujo patógenos progridem rapidamente no organismo, há dificuldade no tratamento pela não resposta imune eficaz do organismo (RAMOS JM, et al., 2017). Essa coinfecção tem várias características, por exemplo, a maior taxa de toxicidade por drogas, menores taxas de cura e maior taxa de recidiva e mortalidade em comparação com Leishmaniose Visceral (LV) na população geral, também pode ocorrer, em alguns casos, a forma visceral crônica ativa, que se caracteriza por sintomas leves e persistentes e uma contínua multiplicação parasitológica mesmo com o tratamento adequado (COTA GF, et al., 2017).

A coinfecção por Leishmania ocasionou uma complicação ainda maior em pacientes infectados com HIV. Como a leishmaniose afeta a função dos monócitos e macrófagos, o surgimento da progressão do HIV é acelerado, assim, há diminuição da expectativa de vida do paciente. Outrossim, o risco de LV clínica aumenta mais de 100 vezes quando o indivíduo é portador de HIV. Vale salientar que pacientes infectados com HIV acabam por desenvolver leishmaniose visceral na presença de algumas espécies de Leishmania, na maioria das vezes, são dermotrópicas (SINGH S, et al., 2014; MARTíNEZ DY, et al., 2018).

HIV e LV dividem mecanismos imunopatológicos semelhantes e afetando as mesmas áreas imunológicas, como células dendríticas (DC) e macrófagos, já citados anteriormente (LINDOSO JA, et al., 2014). Como a proliferação de linfócitos T está afetada pela Leishmania e, por consequência, a produção de interferon gama (INF-c) em pacientes imunocomprometidos, favorece a proliferação e reativação de ambos os patógenos e acelera a progressão do LV e do HIV. Com isso, as duas doenças realizam efeito prejudicial sinérgico no sistema imune, pois seu alvo são células de imunidade semelhantes (ALEMAYEHU M, et al., 2016; LINDOSO JAL, et al., 2016).

Na coinfecção, a imunossupressão do paciente é um fator importante para o desenvolvimento e reativação da LV, quando o paciente apresenta baixa quantidade dos linfócitos T CD4 (LINDOSO JAL, et al., 2016).

A ocorrência de esplenomegalia se difere entre os não coinfectados e os coinfectados, a frequência mais baixa está entre os coinfectados com HIV, isso é explicado por uma falha na resposta imune dos macrófagos. Dentro dos coinfectados, a quantidade de recidivas foi o dobro dos não coinfectados, e a letalidade foi de $25 \%$ nos coinfectados, o triplo quando se compara com os não coinfectados (SOUSA-GOMES ML, et al., 2017; SOUSA-GOMES ML, et al., 2017). 
Cerca de 70\% de casos de coinfecção por Leishmania-HIV na Espanha acometem usuários de drogas intravenosas, isso se explica pelo fato de troca de agulhas, na qual o DNA de Leishmania é encontrado nestas, tendo assim, um ciclo de transmissão antroponótica. Um outro aspecto importante para acontecer essa coinfecção é o deslocamento dessas pessoas da zona urbana para rural, uma migração econômica, na qual já é uma área com grande prevalência de LV (LINDOSO JA, et al., 2014).

$\mathrm{Na}$ associação das duas patologias, as espécies dermotrópicas podem se desenvolver ao sistema reticuloendotelial e em vários outros órgãos, abrangendo as vísceras, mas essas espécies visceralizadoras podem se manifestar de forma atípica. Todas as formas de leishmaniose visceral em indivíduos portadores de HIV devem ser tratadas como uma doença definidora de AIDS em estágio 4, mesmo sendo, segundo a OMS, avaliada em grau de disseminação do HIV e seja mencionado apenas a leishmaniose disseminada atípica (SINGH S, et al., 2014; BURZA S, et al., 2018).

Por ser uma infecção oportunista em pacientes coinfectados por HIV, a Leishmaniose se apresenta constantemente com características clínicas atípicas. Essas manifestações prejudicam o diagnóstico precoce e ocasionam o tratamento tardio nesses pacientes atípicas (ALEMAYEHU M, et al., 2017; LINDOSO JA, et al., 2014).

As manifestações clínicas encontradas na coinfecção LV/HIV se diferem daquelas encontradas nos pacientes não coinfectados. Em relação às diferenças, pacientes coinfectados apresentaram febre inicialmente intermitente seguida por um padrão contínuo de fraqueza, fenômenos hemorrágicos, perda de peso, infecção secundária e tosse quando comparados aos pacientes infectados apenas pela LV, em que esplenomegalia, febre e hepatomegalia foram mais frequentes (SOUSA-GOMES ML, et al., 2017; SILVA LRM, et al., 2018).

Em pacientes com a contagem reduzida de linfócitos CD4+ (<200 células/ $\mu \mathrm{l})$, o médico deve se atentar à presença de Leishmaniose, mesmo na ausência de sinais clássicos, pois podem ser encontradas manifestações atípicas, como o envolvimento do trato gastrointestinal e do trato respiratório (MONGE-MAILLO B, et al., 2014).

As características típicas dos pacientes coinfectados com LV/HIV são, febre de início intermitente seguida de um padrão contínuo; hepatoesplenomegalia não dolorosa, causada pelo envolvimento do sistema reticuloendotelial; pancitopenia, causada pela invasão de parasitas diretamente a medula óssea, provocando sinais e sintomas relacionados a citopenia, gerando anemia, hemorragias e infecções concomitantes. No caso da perda de peso e anorexia, elas podem ser diagnosticadas erroneamente com outras infecções oportunistas (LINDOSO JAL, et al., 2016).

Nas Américas, as características clínicas atípicas foram o acometimento do trato gastrointestinal (pâncreas e duodeno) e do rim, além de fenômenos hemorrágicos (epistaxe, equimoses e hematúria) em cerca de um terço dos pacientes. Segundo a maior série publicada nas Américas, foi observado também o aumento dos linfonodos em $20 \%$ dos pacientes coinfectados, sinal incomum nessa região e comum no Mediterrâneo (LINDOSO JA, et al., 2014).

É incomum o envolvimento cutâneo na coinfecção entre LV/HIV e, na América Latina, raramente são relatadas lesões cutâneas provocadas por $L$. infantum. A Leishmaniose causada por $L$. infantum já foi mencionada com Leishmaniose cutânea associada simultaneamente. Outras pesquisas indicam a possibilidade de visceralização da infecção por Leishmaniose cutânea, devido a anteriorização dela em relação ao acometimento visceral. Lesões mucocutâneas por L. infantum já foram citadas em pacientes HIV positivos e a presença de Leishmania em lesões cutâneas já levou ao diagnóstico de Leishmaniose visceral. No entanto, a presença do parasita em doenças sem etiologia estabelecida, pode demonstrar presença passiva devido à disseminação em pacientes imunologicamente comprometidos. Além disso, também foram citadas a Leishmaniose dérmica pós-calazar (PKDL) por L. infantum, a Leishmaniose cutânea disseminada atípica com lesões maculopapulares, difusas ou não ulceradas após a doença visceral e a presença de uveíte em associação com PKDL em pacientes coinfectados (LINDOSO JA, et al., 2014; MONGE-MAILLO B, et al., 2014). 
No que se refere à evolução clínica da infecção por HIV e LV, ambas são demasiadamente dependentes da resposta imune específica e das alterações do sistema imunológico causadas por ambos os patógenos, sendo que o desenvolvimento de ambas as doenças pode ser acelerado devido à coinfecção (LINDOSO JA, et al., 2014).

A predisposição à recaída nesses pacientes é uma das características mais observadas nos estudos de coinfecção LV/HIV. Nesse contexto, o tratamento desses pacientes pode se tornar difícil, uma vez que normalmente possuem altas taxas de mortalidade e baixas taxas de cura em relação a pacientes HIVnegativos, mais recaídas, mais falhas de tratamento, maior toxicidade e resistência aos compostos antimoniais pentavalentes (SINGH S, et al., 2014).

Além disso, a presença de obstáculos, como o alto custo, a baixa capacidade de produção, a geografia física e problemas no controle de qualidade impossibilitam o acesso aos poucos tratamentos disponíveis (SOUSA-GOMES ML, et al., 2017; COTA GF, et al., 2011; MONGE-MAILLO B, et al., 2014; DIRO E, et al., 2019; LOUIS L, et al., 2019).

Como citado previamente, a coinfecção por Leishmaniose-HIV apresenta algumas dificuldades na identificação das manifestações clínicas atípicas, seja em formas viscerais ou tegumentares e no diagnóstico (LINDOSO JAL, et al., 2016).

O tratamento de escolha da coinfecção Leishmania/HIV é a anfotericina lipossomal, pois é a que apresenta melhores resultados nesses pacientes. No Brasil, essa droga é recomendada pelo Ministério da Saúde (MS) e pela OMS. No Mediterrâneo, a terapia antirretroviral na população proporcionou a redução da incidência entre 50-65\%, além de promover o aumento das taxas de sobrevida e redução de recaídas e possíveis síndromes inflamatórias de reconstituição imunológica. Contudo, ainda não existe um consenso sobre a dosagem de anfotericina lipossomal e a melhor opção de tratamento para a população do mundo todo (SILVA LRM, et al., 2018; LINDOSO JAL, et al., 2016; WHO, 2010).

O uso da terapia antirretroviral altamente ativa (HAART) mostrou melhora significativa na sobrevivência dos coinfectados LV/HIV, entretanto, continua a preocupação relacionada a altas taxas de recidiva e casos da "forma visceral crônica ativa", a qual se caracteriza pela contínua multiplicação parasitológica mesmo com o tratamento adequado. Ademais, o uso do HAART não impactará na população com difícil acesso ao sistema de saúde, como os pobres que vivem na periferia das grandes cidades e desconhecem sua sorologia para o HIV (COTA GF, et al., 2017; SOUSA-GOMES ML, et al., 2017).

Estudos mostraram que o uso do HAART e profilaxia secundária (para pacientes com contagem de linfócitos CD4 inferior a 350 células $/ \mathrm{mm} 3$ ), resultaram em melhora clínica nos pacientes, com a eliminação da febre associada a um desses achados: redução de $2 \mathrm{~cm}$ ou mais na palpação do baço realizada pelo menos examinador, aumento de $2 \mathrm{~g} \%$ ou mais do nível da hemoglobina, aumento de $50 \%$ ou mais na contagem de leucócitos e aumento de 50\% ou mais na a contagem de plaquetas (COTA GF, et al., 2017).

Pacientes imunossuprimidos gravemente com a presença de doenças oportunistas, além do uso do HAART, podem ter problemas de interação medicamentosa. Resumidamente, durante o tratamento de infecções oportunistas e AIDS, a interação medicamentosa é generalizada (LINDOSO JAL, et al., 2016).

\section{CONSIDERAÇÕES FINAIS}

A partir do trabalho é possível constatar que a coinfecção HIV/Leishmaniose se mostra uma patologia com intenso poder ofensivo ao sistema imune, uma vez que ambas as doenças realizam efeito prejudicial sinérgico a esse sistema, o que gera dificuldade no tratamento pela resposta imune não eficaz do organismo. Ainda mais, soma-se ao impasse do tratamento alguns fatores como as recaídas e a maior taxa de toxicidade por drogas, situação essa que favorece a redução da sobrevida do paciente. Além disso, foi possível verificar que as manifestações clínicas presentes na coinfecção, embora possuam apresentação diferente quando comparadas à infecção isolada, que a presença de características atípicas ou a ausência de características típicas, dificulta o diagnóstico da doença. 


\section{REFERÊNCIAS}

1. ABONGOMERA C, et al. Development and external validation of a clinical prognostic score for death in visceral leishmaniasis patients in a high HIV coinfection burden area in Ethiopia. PLoS ONE, 2017, 12(6).

2. ALEMAYEHU M, et al. Magnitude of visceral leishmaniasis and poor treatment outcome among HIV patients: metaanalysis and systematic review. Research and Palliative Care, 2016; 8: 75-81.

3. ALEMAYEHU M, et al. Prevalence of Human Immunodeficiency Virus and associated factors among Visceral Leishmaniasis infected patients in Northwest Ethiopia: a facility based cross sectional study. BMC Infectious Diseases, 2017; 17: 152.

4. BURZA S, et al. Leishmaniasis. Seminar, 2018; 392: 951-970.

5. COTA GF, et al. Exploring prognosis in chronic relapsing visceral leishmaniasis among HIV-infected patients: Circulating Leishmania DNA. Acta Tropica, 2017; 172: 186-191.

6. COTA GF, et al. Predictors of Visceral Leishmaniasis Relapse in HIV Infected Patients: A Systematic Review. PLoS Neglected Tropical Diseases, 2011; 5(6).

7. DIRO $\mathrm{E}$, et al. Long term outcomes and prognostics of visceral leishmaniasis in hiv infected patients with use of pentamidine as secondary prophylaxis based on CD4 level: a prospective cohort study in Ethiopia. PLoS neglected tropical diseases, 2019; 13(2).

8. LEELAYOOVA S, et al. Leishmaniasis in Thailand: a review of causative agents and situations. Am. J. Trop. Med. Hyg., 2017; 96(3): 534-542

9. LINDOSO JA, et al. Visceral Leishmaniasis and HIV Coinfection in Latin America. PLoS Neglected Tropical Diseases, 2014; 8(9).

10. LINDOSO JAL, et al. Leishmaniasis-HIV coinfection: Current challenges HIV/AIDS. Research and Palliative Care, 2016; 8: 147-156.

11. LOUIS L, et al. Intradermal Synthetic DNA Vaccination Generates Leishmania Specific T Cells in the Skin and Protection against Leishmania major. Infection and Immunity, 2019, 87(8).

12. MARTÍNEZ DY, et al. Tegumentary leishmaniasis and coinfections other than HIV. PLoS Neglected Tropical Diseases, 2018; 12(3): 1-20.

13. MARTINS GAS, LIMA MD. Leishmaniose: Do diagnóstico ao tratamento. Enciclopédia biosfera, 2013; 9(6): 2556.

14. MEIRELES CB, et al. Atypical presentations of cutaneous leishmaniasis: A systematic review. Acta Tropica, 2017; $172(3): 240-254$.

15. MONGE-MAILLO B, et al. Visceral Leishmaniasis and HIV Coinfection in the Mediterranean Region. PLOS Neglected Tropical Diseases, 2014; 8(8).

16. RAMOS JM, et al. Is visceral leishmaniasis different in immunocompromised patients without human immunodeficiency virus? A comparative, multicenter retrospective cohort analysis. American Journal of Tropical Medicine and Hygiene, 2017; 97(4): 1127-1133.

17. SALOMÃO R. Infectologia - Bases Clínicas e Tratamento. Rio de Janeiro: Guanabara Koogan, 2017; 644p.

18. SILVA LRM, et al. Distribuição espacial da coinfecção leishmaniose visceral-HIV em Teresina/PI, 2006 a 2015.Tese (Mestrado em Saúde Coletiva) - Centro Biomédico, Instituto de Medicina Social. Universidade do Estado do Rio de Janeiro, Rio de Janeiro, 2018; 63 p.

19. SINGH S, et al. Changing trends in the epidemiology, clinical presentation, and diagnosis of Leishmania-HIV coinfection in India. International Journal of Infection Diseases, 2014; 29: 103-112.

20. SOUSA-GOMES ML, et al. Visceral leishmaniasis and HIV/AIDS in Brazil: Are we aware enough? PLoS Neglected Tropical Diseases, 2017; 11(9): 1-13.

21. WHO. Control of the Leishmaniases. World Health Organization, 2010; 949(3). 